Clinical Research Paper

\title{
Better pathologic complete response and relapse-free survival after carboplatin plus paclitaxel compared with epirubicin plus paclitaxel as neoadjuvant chemotherapy for locally advanced triple-negative breast cancer: a randomized phase 2 trial
}

\author{
Pin Zhang ${ }^{1, *}$, Yi Yin ${ }^{2}$, Hongnan Mo ${ }^{1}$, Bailin Zhang ${ }^{3}$, Xiang Wang ${ }^{3}$, Qing Li ${ }^{1}$, Peng \\ Yuann ${ }^{1}$, Jiayu Wang ${ }^{1}$, Shan Zheng ${ }^{4}$, Ruigang $\mathrm{Ca}^{1}{ }^{1}$, Fei Ma ${ }^{1}$, Yin Fan ${ }^{1}$ and Binghe $\mathrm{Xu}^{1, *}$ \\ ${ }^{1}$ Department of Medical Oncology, Cancer Hospital, Chinese Academy of Medical Sciences and Peking Union Medical College, \\ Beijing, China \\ 2 Department of Medical Oncology, Fujian Provincial Cancer Hospital, Fuzhou, China \\ ${ }^{3}$ Department of Breast Surgery, Cancer Hospital, Chinese Academy of Medical Sciences and Peking Union Medical College, \\ Beijing, China \\ ${ }^{4}$ Department of Pathology, Cancer Hospital, Chinese Academy of Medical Sciences and Peking Union Medical College, \\ Beijing, China \\ * These authors have contributed equally to the work \\ Correspondence to: Binghe Xu, email: bhxu@hotmail.com \\ Keywords: breast cancer; triple negative; neoadjuvant chemotherapy; carboplatin; paclitaxel \\ Received: April 02, $2016 \quad$ Accepted: June 13, $2016 \quad$ Published: July 14, 2016
}

ABSTRACT

Background: No standard chemotherapy is used as neoadjuvant therapy in triple negative breast cancer (TNBC). This study has compared carboplatin plus paclitaxel with commonly used epirubicin plus paclitaxel as neoadjuvant chemotherapy (NAC) in TNBC.

Results: 91 patients with a median age of 47 years (PC 47 patients, EP 44 patients) were enrolled. $65 \%$ of the patients were premenopausal. While the objective response rate was similar in the PC and EP arm (89.4\% vs. $79.5 \%, P=0.195)$, the PCR rate in the PC arm was significantly higher $(38.6 \%$ vs. $14.0 \%, P=0.014)$.

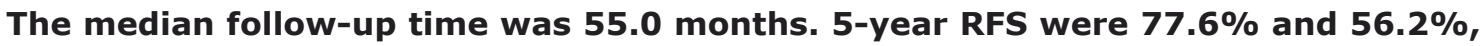
significantly higher in the PC arm, $P=0.043$. No significant difference in OS was observed between the two arms $(P=0.350)$. Adverse events were similar, except for more thrombocytopenia in the PC arm $(P=0.001)$.

Methods: Patients with stage II/III TNBC were randomized to receive either paclitaxel $\left(175 \mathrm{mg} / \mathrm{m}^{2}\right.$, day1) plus carboplatin (Area Under the Curve = 5, day2) (PC) or epirubicin $\left(75 \mathrm{mg} / \mathrm{m}^{2}\right.$, day1) plus paclitaxel $\left(175 \mathrm{mg} / \mathrm{m}^{2}\right.$, day 2$)(E P)$ as NAC every three weeks for 4-6 cycles. The primary endpoint was rate of pathologic complete response (PCR).The secondary endpoints included relapse-free survival (RFS), overall survival (OS) and safety.

Conclusions: This study suggested that the addition of carboplatin to paclitaxel was superior to the regimen of epirubicin plus paclitaxel as NAC for TNBC in terms of improving PCR rate and RFS. Further phase 3 study has already started.

\section{INTRODUCTION}

Triple-negative breast cancer (TNBC), defined as the lack of expression of estrogen receptor (ER), progesterone receptor (PR), and human epidermal growth factor receptor 2 (HER2), accounts for approximately $20 \%$ of all breast cancers [1,2]. Without specific treatment such as endocrine therapy or anti-HER 2 therapy for this subtype of breast cancer, cytotoxic chemotherapy remains the only choice of treatment [2].

Neoadjuvant chemotherapy (NAC) with both anthracycline and taxane combination resulted in higher 
Table 1: Patient characteristics at baseline (intention-to-treat population)

\begin{tabular}{|l|l|l|l|}
\hline Characteristics & PC arm $(\boldsymbol{N}=\mathbf{4 7})$ & EP arm $(\mathbf{N}=\mathbf{4 4})$ & $\boldsymbol{P}$ value \\
\hline Age, median years (range) & $48(24-73)$ & $46(24-65)$ & 0.205 \\
\hline Menopausal status & & & 0.518 \\
\hline Premenopausal & $29(61.7 \%)$ & $30(68.2 \%)$ & \\
Postmenopausal & $18(38.3 \%)$ & $14(31.8 \%)$ & \\
\hline Clinical tumor stage & & & 0.271 \\
\hline T1 & $1(2.13 \%)$ & $4(9.09 \%)$ & \\
T2 & $24(51.6 \%)$ & $25(56.82 \%)$ & \\
T3 & $13(27.66 \%)$ & $16(36.36 \%)$ & \\
T4 & $9(19.15 \%)$ & $6(13.64 \%)$ & 0.055 \\
\hline Clinical nodal stage & & & \\
\hline N0 & $13(27.66 \%)$ & $8(18.18 \%)$ & \\
N1 & $13(27.66 \%)$ & $21(47.73 \%)$ & \\
N2 & $17(36.17 \%)$ & $8(15.91 \%)$ & 1.000 \\
N3 & $4(8.51 \%)$ & & \\
\hline Clinical stage & & $15(34.09 \%)$ & \\
\hline II & $16(34.04 \%)$ & $29(65.91 \%)$ & \\
III & $31(65.96 \%)$ & & 0.054 \\
\hline Ki-67 & & $12(33.33 \%)$ & \\
\hline$<20 \%$ & $5(13.16 \%)$ & $24(66.67 \%)$ & \\
\hline 20\% & $33(86.84 \%)$ & $33(81.67 \%)$ & \\
\hline CK5/6, EGFR & & & \\
\hline Either positive & $36(97.30 \%)$ & & \\
Both negative & $1(2.70 \%)$ & & \\
\hline
\end{tabular}

Abbreviation: PC, paclitaxel plus carboplatin regimen; EP, epirubicin plus paclitaxel regimen; CK5/6, Cytokeratin 5/6; EGFR, epidermal growth factor receptor.

Data are n (\%) unless stated otherwise.

pathologic complete response (pCR) rate $(28 \%)$ than those of anthracycline-based $(20 \%)$ or taxane-based $(12 \%)$ regimens [3]. It is believed to be the most effective regimen for $\mathrm{TNBC}$ in neoadjuvant setting. A pCR rate of $38.9 \%$ was observed after anthracycline-taxane-based NAC in patients with operable or locally advanced TNBC [4]. Although there was strong association between higher pCR rate and improved relapse-free survival (RFS) and overall survival (OS), the overall prognosis of TNBC remains poor including high risk of early recurrence involving viscera or central nervous system $[1,5,6]$. Data from M.D. Anderson Cancer Center showed that the 5 -year RFS and OS rates were $61 \%$ and $64 \%$ after NAC in patients with operable or locally advanced TNBC [3]. The 7-year RFS and OS rates were only $57 \%$ and $65 \%$ in locally advanced TNBC after anthracycline-taxanecyclophosphamide NAC [7].

The role of platinum-based NAC has been investigated in TNBC and was gradually brought to attention during the past few years. Results from two randomized phase II studies suggested that in TNBC, the addition of carboplatin to anthracycline-taxanebased NAC significantly improved the pCR rate, but resulted in more toxicity-related delay of treatment [8, 9]. Unfortunately, neither study has reported relapse-free survival and overall survival due to lack of long term follow up. Hurly et al retrospectively analyzed docetaxel plus carboplatin as anthracycline-free NAC in 27 locally advanced TNBC patients resulting in a pCR rate of $26 \%$ [10]. Shindle et al retrospectively reported a pCR rate of $60 \%$ and a 25 -months of median relapse-free survival in 10 locally advanced TNBC patients treated by paclitaxel plus carboplatin NAC. [11].

To date, no prospective or randomized study has compared a platinum-based regimen to commonly used anthracycline-taxane regimens in TNBC in neoadjuvant setting. This randomized controlled phase II study is designed to compare paclitaxel plus carboplatin given as NAC with epirubicin plus paclitaxel in locally advanced TNBC.

\section{RESULTS}

\section{Patient characteristics}

Between May 2006 and December 2012, 91 patients were enrolled and started study treatment. 47 patients with PC regimen and 44 patients with EP regimen were included in the intention-to-treat and safety populations (Figure 1).

Baseline characteristics of the 91 treated patients are listed in Table 1. Median age was 47 years (range 24-73 
Table 2: Hematological and non-hematological adverse events

\begin{tabular}{|c|c|c|c|c|c|c|}
\hline \multirow[b]{2}{*}{ Adverse events } & \multicolumn{2}{|c|}{ PC $\operatorname{arm}(N=47)$} & \multicolumn{2}{|c|}{ EP $\operatorname{arm}(N=44)$} & \multicolumn{2}{|l|}{$P$ value } \\
\hline & All grade & Grade 3/4 & All grade & Grade 3/4 & All grade & Grade 3/4 \\
\hline \multicolumn{7}{|c|}{ Hematological toxicity } \\
\hline Neutropenia & $43(91.5 \%)$ & $34(72.3 \%)$ & $37(84.1 \%)$ & $28(63.6 \%)$ & 0.344 & 0.500 \\
\hline Thrombocytopenia & $17(36.2 \%)$ & $4(8.5)$ & $2(4.5)$ & 0 & 0.001 & 0.118 \\
\hline \multicolumn{7}{|c|}{ Non-hematological toxicity } \\
\hline Vomiting & $41(87.2 \%)$ & $1(2.1 \%)$ & $36(81.8 \%)$ & $4(9.1 \%)$ & 0.567 & 0.194 \\
\hline $\begin{array}{l}\text { Peripheral } \\
\text { neuropathy }\end{array}$ & $19(40.4 \%)$ & 0 & $17(38.6 \%)$ & 0 & 1.000 & - \\
\hline ALT/AST elevation & $15(31.9 \%)$ & 0 & $14(31.8 \%)$ & 0 & 0.992 & - \\
\hline Myalgia/arthralgia & $10(21.3 \%)$ & 0 & $8(18.2 \%)$ & 0 & 0.715 & - \\
\hline ST-T changes & $9(19.2 \%)$ & 0 & $11(25.0 \%)$ & 0 & 0.495 & - \\
\hline
\end{tabular}

Abbreviation: PC, paclitaxel plus carboplatin regimen; EP, epirubicin plus paclitaxel regimen.

Data are n (\%) unless stated otherwise.

years). All enrolled patients were female, $65 \%$ of whom were premenopausal. More than half had T2 tumors. $76.9 \%$ of all patients were clinically node positive. More than two-thirds of patients had Ki-67 proliferation index $>$ $20 \%$. Notably, $94.4 \%$ of patients were positive for either CK $5 / 6$ or EGFR, $97.30 \%$ in PC arm and $91.67 \%$ in the EP arm, respectively $(P=0.358)$. Baseline characteristics were well balanced between two arms.

87 patients underwent surgery and had tumor responses evaluated pathologically. 75 patients underwent modified radical mastectomy and 12 patients had breastconserving surgery. 79 patients received at least 4 cycles of NAC, and 38 patients completed six cycles of NAC. 4 patients in PC arm switched to anthracycline-based adjuvant chemotherapy, and 13 patients in EP arm switched to platinum-based regimen after surgery due to lack of objective response in NAC. 23 patients in the PC arm and 21 patients in the EP arm received post-operative radiotherapy. All the patients who underwent breastconserving surgery received radiotherapy.

\section{Clinical efficacy}

Of the 91 patients who were evaluated for clinical response, the overall ORR was similar between the two regimens $(P=0.195): 89.4 \%$ in $\mathrm{PC}$ arm and $79.5 \%$ in EP arm, respectively. 87 patients underwent surgery and had tumor responses evaluated pathologically. 23 of 87 patients $(26.4 \%)$ had pCR (ypT0/isN0) of the invasive breast cancer both in breast and axilla, including 17 patients in PC arm and 6 patients in EP arm. The pCR (ypT0/isN0) rate in the PC arm was significantly higher compared to the EP arm $(38.6 \%$ vs. $14.0 \%$; risk ratio $=$ 3.876; $P=0.014$; Figure 2). Furthermore, $18 / 44$ patients $(43.2 \%)$ in $\mathrm{PC}$ arm and $8 / 43$ patients $(18.60 \%)$ in EP arm had pCR (ypT0/is) in breast $(P=0.024)$, whereas $20 / 32$ patients $(62.5 \%)$ in $\mathrm{PC}$ arm and $10 / 34$ patients $(29.4 \%)$ in
EP arm had pCR (ypN0) in axilla $(P=0.008)$.

Figure 3 illustrates the subgroup analysis in patients achieving pCR after two different treatments. The pCR rate for PC was numerically superior in all subgroups, but this difference reached statistical significance only in premenopausal women, clinically evaluated lymph nodes and patients with stage III disease.

\section{Survival analysis}

The cut-off date for survival analysis was November $9^{\text {th }}, 2015$. Median follow-up time was 55.0 months (4.0-105.0 months). Median OS has not been reached. During the study period, a total of 27 relapse events were recorded, 9 in the $\mathrm{PC}$ arm and 18 in the EP arm. Most events $(88.9 \%)$ were observed during the first 3 years after first diagnosis. The most common site of recurrence was lung and lymph node: $4(44.4 \%)$ and $5(55.6 \%)$ patients in the PC arm, and 12 (66.7\%) and 7 (38.9\%) patients in the EP arm, respectively. The post-recurrence survival for the entire study population was $17.7 \pm 5.6$ months, with no significant difference between the two arms (PC arm, $12.1 \pm 3.8$ months; EP arm, $18.7 \pm 9.1$ months; $P=0.445)$. In the PC arm, there were 7 deaths from any cause, 2 of which died of stroke. Eleven patients died in the EP arm, all of whom were cancer-related deaths.

Figures 4 shows the Kaplan-Meier estimates of RFS and OS. Patients in the PC arm had significantly higher RFS rate than that in the EP $\operatorname{arm}(P=0.043)$. The 1-, 3-, and 5-year RFS rate were $93.0 \%, 81.2 \%$, and $77.6 \%$ in the PC arm, and $85.7 \%, 61.6 \%$, and $56.2 \%$ in the EP arm, respectively. From another point of view, patients who achieved pCR had significantly improved RFS $(P=0.001)$ compared with those with residual disease after NAC. In 23 patients with pCR, only one of them had disease recurrence. Relapse occurred in the lung 46 months after diagnosis (42 months after surgery), and the patient was 
still alive after 95.0 months' follow-up. The 5-year RFS rate were $94.7 \%$ for patients achieving pCR, and $56.1 \%$ for patients with residual disease.

As to the OS, there was no significant difference between the PC regimen and EP regimen $(P=0.350)$. The 1-, 3-, and 5-year OS rate were $100.0 \%, 85.8 \%$, and $83.3 \%$ in the PC arm; and $95.2 \%, 83.2 \%$, and $70.7 \%$ in the EP arm, respectively. Patients who achieved pCR had significantly improved OS $(P=0.004)$ compared with those with residual disease after NAC. The 5-year
OS rate were $100.0 \%$ for pCR patients, and $67.2 \%$ for patients with residual disease, respectively. In patients with residual disease, those who received $\mathrm{PC}$ regimens had similar RFS $(P=0.217)$ and OS $(P=0.970)$ compared with those of patients in EP arm.

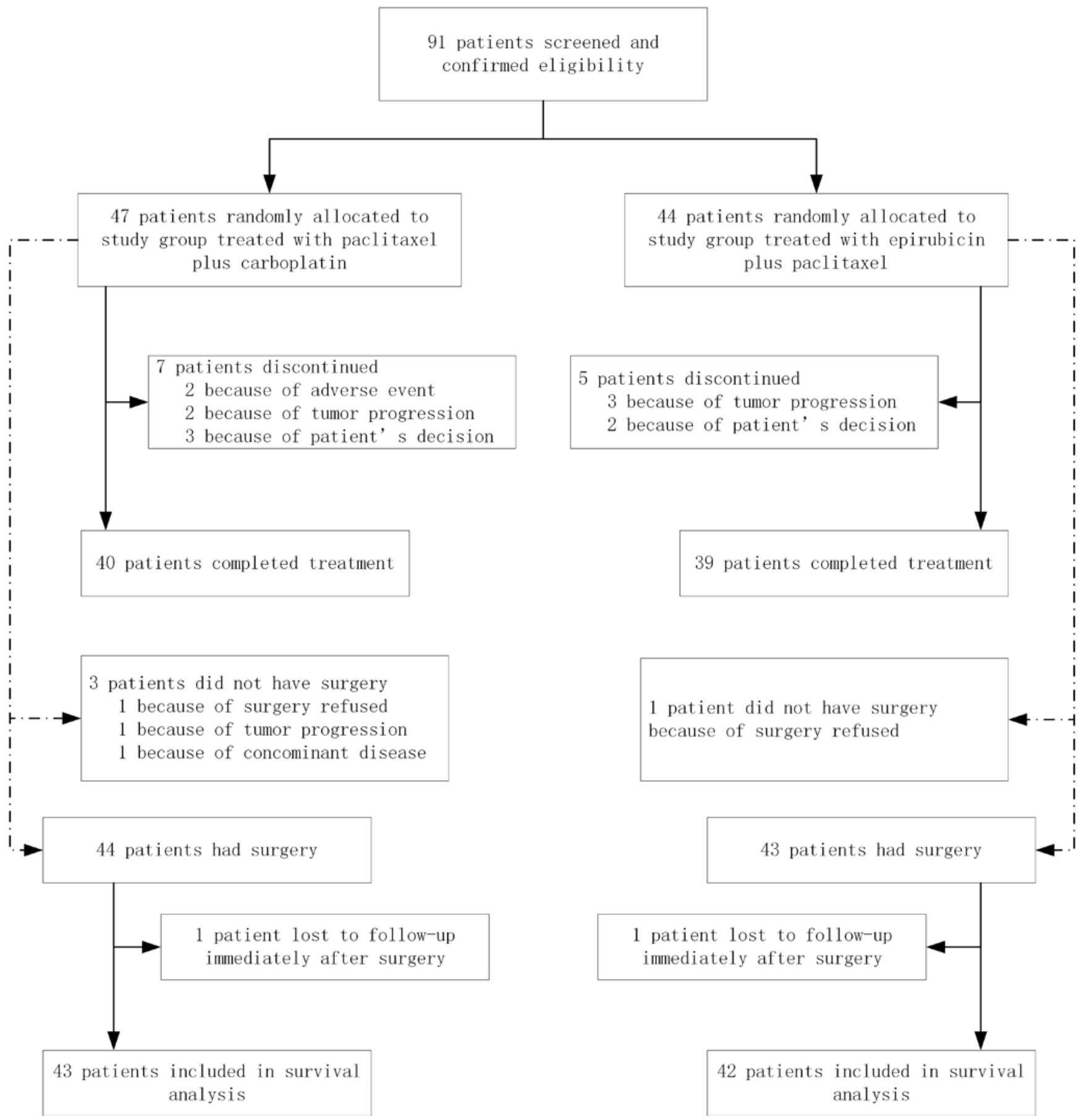

Figure 1: Trial profile. 


\section{NAC safety}

Table 2 lists the adverse events. The most common adverse events were neutropenia and vomiting, similar in the two arms. Grade 3/4 neutropenia resulted in treatment delay or dose reduction for 3 patients $(6.3 \%)$ in the PC arm and 7 patients $(15.9 \%)$ in the EP arm $(P=0.188)$. Thrombocytopenia occurred more commonly in the PC arm. Grade 3/4 thrombocytopenia was present in 4 patients $(8.5 \%)$ in the PC arm, resulting in 2 cases of treatment termination. Other adverse events included peripheral neuropathy, ALT/AST elevation, electrocardiographic ST-T changes and myalgia/arthralgia. No death or lifethreatening event was recorded during the study.

\section{DISCUSSION}

Two randomized phase II studies, CALGB40603 and GeparSixto, have demonstrated significant increases in $\mathrm{pCR}$ rate with the addition of carboplatin to anthracyclinetaxane-based NAC in TNBC (54\% vs. 41\%; $57 \%$ vs. 43\%) $[8,9]$. In both studies, however, the addition of carboplatin was associated with higher incidence of hematological toxicity, dose adjustments, and treatment discontinuations. In another phase II study (GEICAM/2006-03), it was reported carboplatin did not even improve pCR rate $(30 \%$ vs. 35\%) [12]. Furthermore, due to the lack of long-term follow up in these studies, whether higher $\mathrm{pCR}$ rates with carboplatin will improve long-term outcomes such as RFS and OS remains unknown.

Our study is the first prospective, randomized, controlled study showing significant improvements in pCR rate and RFS by anthracycline-free platinum-based NAC in TNBC. A long follow-up time also allowed us to report overall survival in these patients.

We found that in the NAC of TNBC patients, the addition of carboplatin to paclitaxel was superior to the regimen of epirubicin plus paclitaxel in terms of $\mathrm{pCR}$ rate and RFS. This may due to more frequent deficiencies in the BRCA associated DNA repairing mechanism in TNBC $[2,13,14]$. This was already supported by several studies demonstrating a high level activity of platinum as NAC in BRCA-deficient breast cancers $[15,16]$. Telli et al reported that the combination of gemcitabine, carboplatin, and iniparib as NAC in early stage breast cancer resulted in a $\mathrm{pCR}$ rate of $33 \%$ in wild type BRCA $1 / 2,47 \%$ in BRCA $1 / 2$ mutation carriers, and $56 \%$ in BRCA1/2 mutation carriers

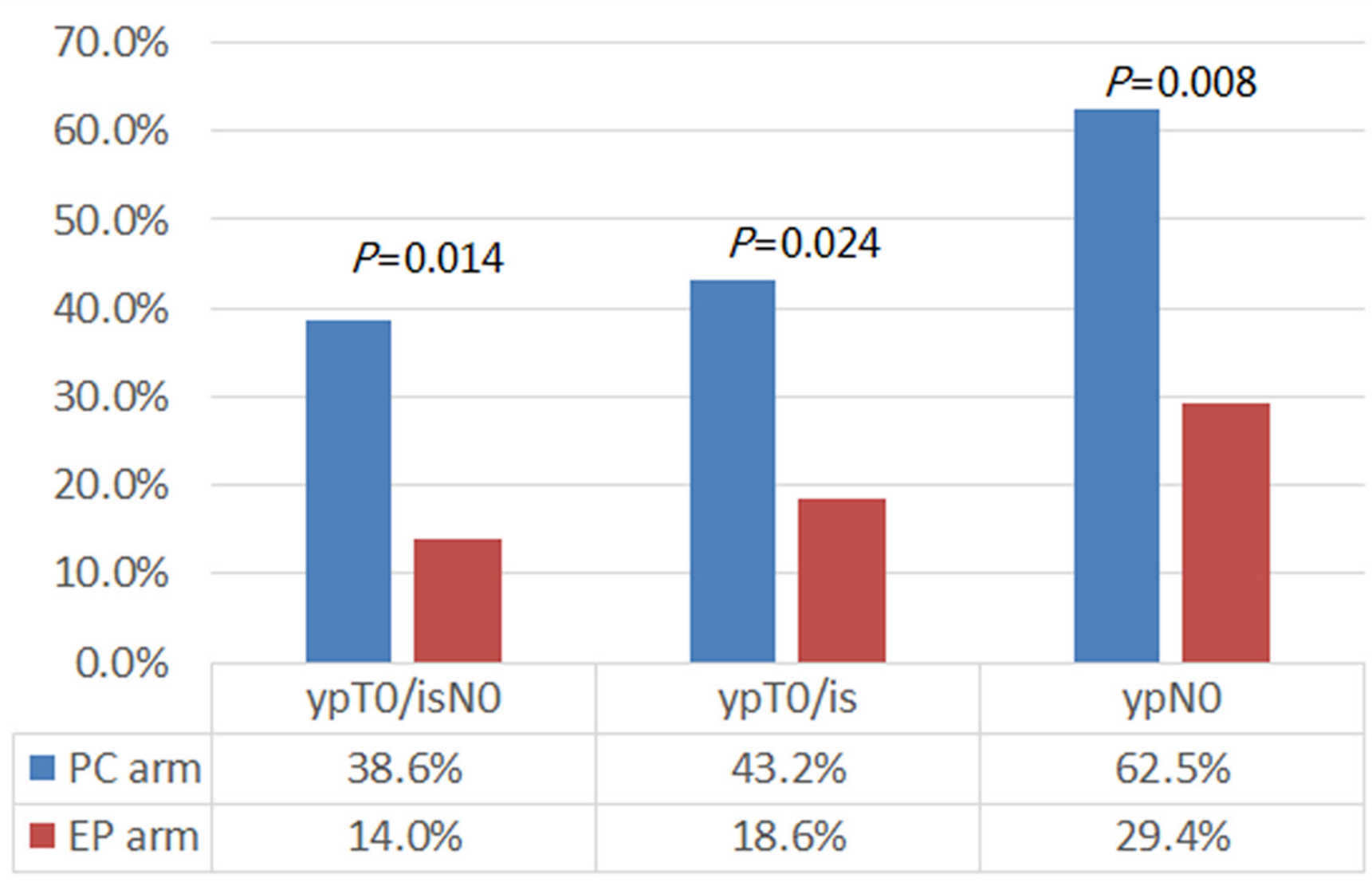

Figure 2: The pathologic complete response (pCR) rate of patients in different arms. When compared with those in the EP arm, patients in the PC arm had significantly higher pCR (ypT0/isN0) rate (38.6\% vs. $14.0 \% ; P=0.014$ ), both in breast (ypT0/is; $43.2 \%$ vs. $18.60 \% ; P=0.024)$ and in axilla (ypN0; $62.5 \%$ vs. $29.4 \% ; P=0.008)$. 
with TNBC [15]. In another study, neoadjuvant cisplatin given every three weeks resulted in a pCR rate of $61 \%$ in TNBC with BRCA1 mutation [16]. Unfortunately, BRCA mutation status was not assessed in this study because of economic and technical conditions at that time. Further comparison of PC and EP as NAC has been planned in breast cancer patients with BRCA-mutations.

Though patients in the $\mathrm{PC}$ arm achieved higher rate in pCR and longer RFS than those in EP arm, it should be noted in patients with residual disease after NAC, RFS was similar between the two arms. We may speculate that the improvement in RFS by PC regimen may be directly linked to the increase in pCR rate after NAC. Additionally, in the overall population, patients who obtained pCR had improved long term survival in the study, a finding consistent with previous reports [6]. The RFS inequality might decline because of the switching between PC and EP regimen in the adjuvant setting. Even so, our study has clearly demonstrated the significant improvement of PC over EP in RFS, probably due to the impact of pCR on RFS.

At the time of this report, the median OS has not been reached, which might partly explain the little difference in OS between two arms. Because of the limited sample size and the small number of deaths, our study is unable to detect an OS difference. Other than the duration of follow-up or subsequent treatment upon recurrence, there are still a number of factors that may impact overall survival. Therefore, RFS seems to be a more appropriate indicator than OS here on whether patients have received direct benefit from the treatment.

It is noticed that the $\mathrm{pCR}$ rate achieved in our study is lower than previous reports [8, 9, 11]. This may due to the robust triplet regimen, paclitaxel and an anthracycline in addition to carboplatin, used in those studies. Besides a averagely higher tumor stage of patient population, this could also be explained by an every three weeks dosing schedule of paclitaxel used in the study, a dosing schedule later largely replaced by a weekly dosing of paclitaxel because of its higher activity and lower toxicity $[8,17,18]$. When our study started in 2006 , the priority of weekly schedule was not definite, and the cost of a weekly schedule of paclitaxel was not covered by patients' medical insurance. That is the reason why we chose 3-weekly schedule of paclitaxel as part of the NAC regimens. Additionally, approximately $95 \%$ of our patients were positive in CK5/6 or EGFR biomarkers associated with basal like genotype and poor survival [19-22]. This would also contribute to the lower pCR rate than that reported in other literature.

In summary, this study supports the addition of carboplatin to NAC for TNBC. Anthracycline-free

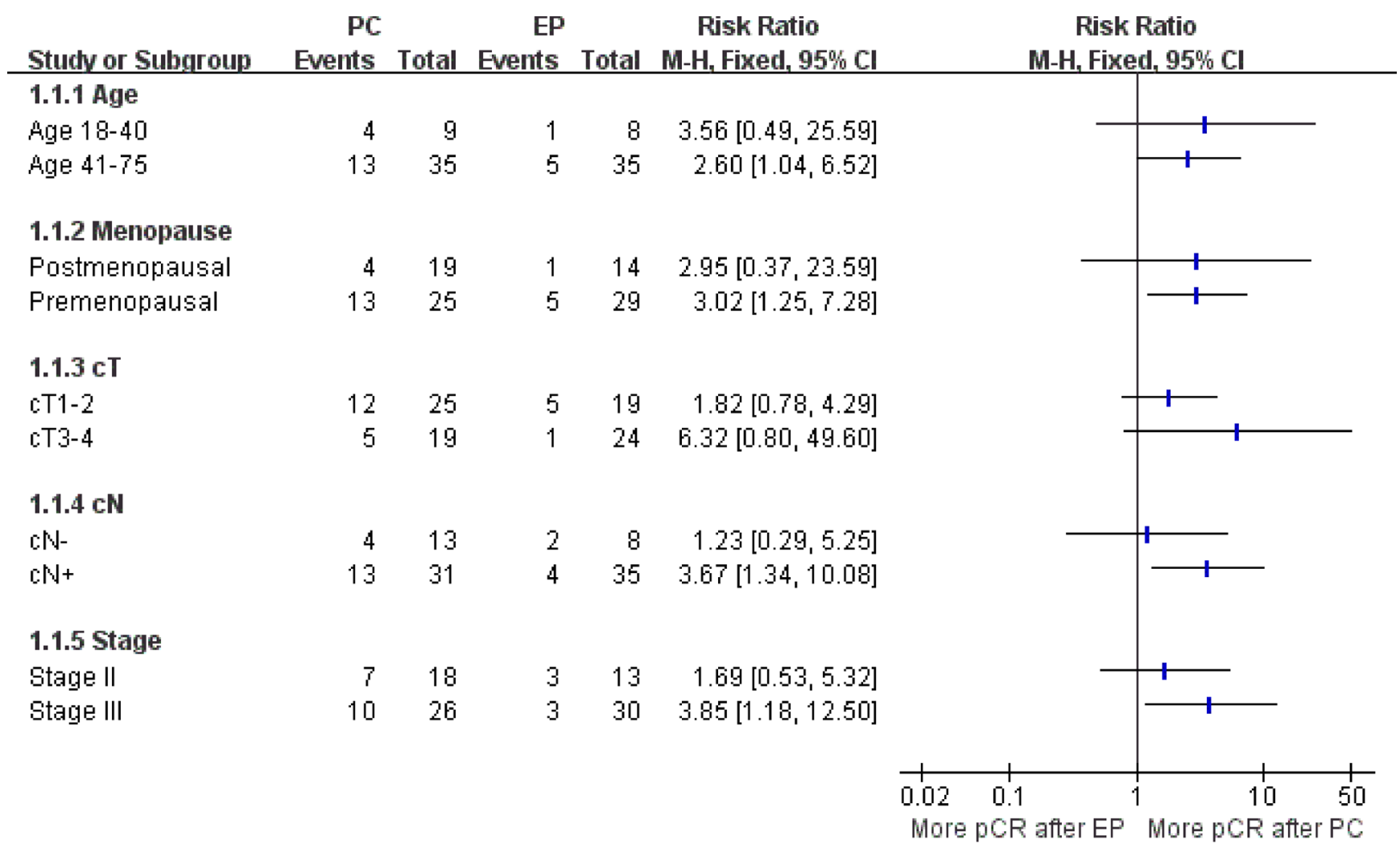

Figure 3: Subgroup analysis in patients achieving pCR after two different treatments. PC, paclitaxel plus carboplatin regimen; EP, epirubicin plus paclitaxel regimen; pCR, pathologic complete response. 
carboplatin-based regimen not only significantly improve pCR rate and RFS but also reduce toxicity, compared to CALGB40603 and GeparSixto studies with concurrent or sequential carboplatin and anthracycline. It is believed
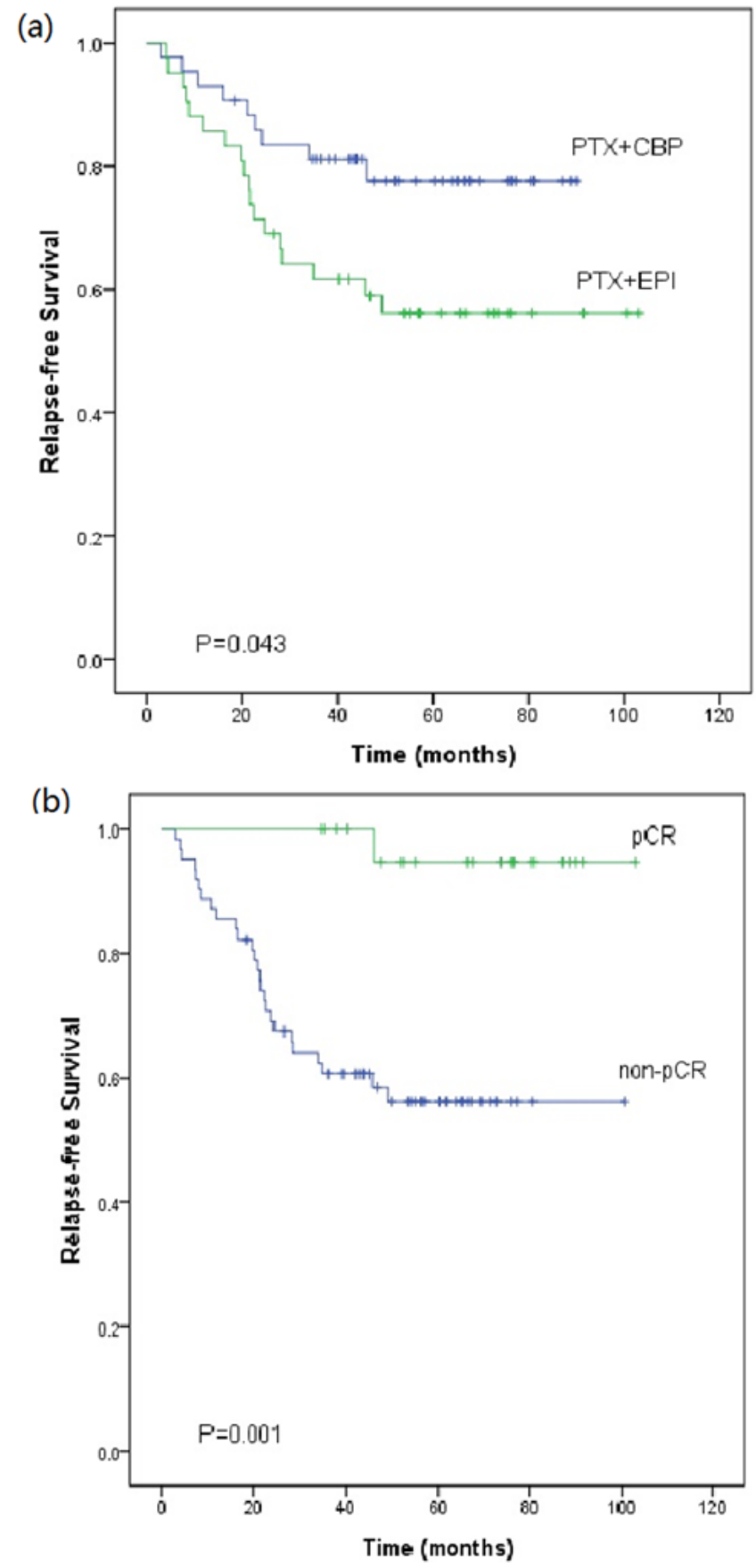

carboplatin plus paclitaxel could be an alternative or even a better neoadjuvant chemotherapy option in TNBC. A phase III study adopting dose intense schedule and identifying potential advantageous subgroups with
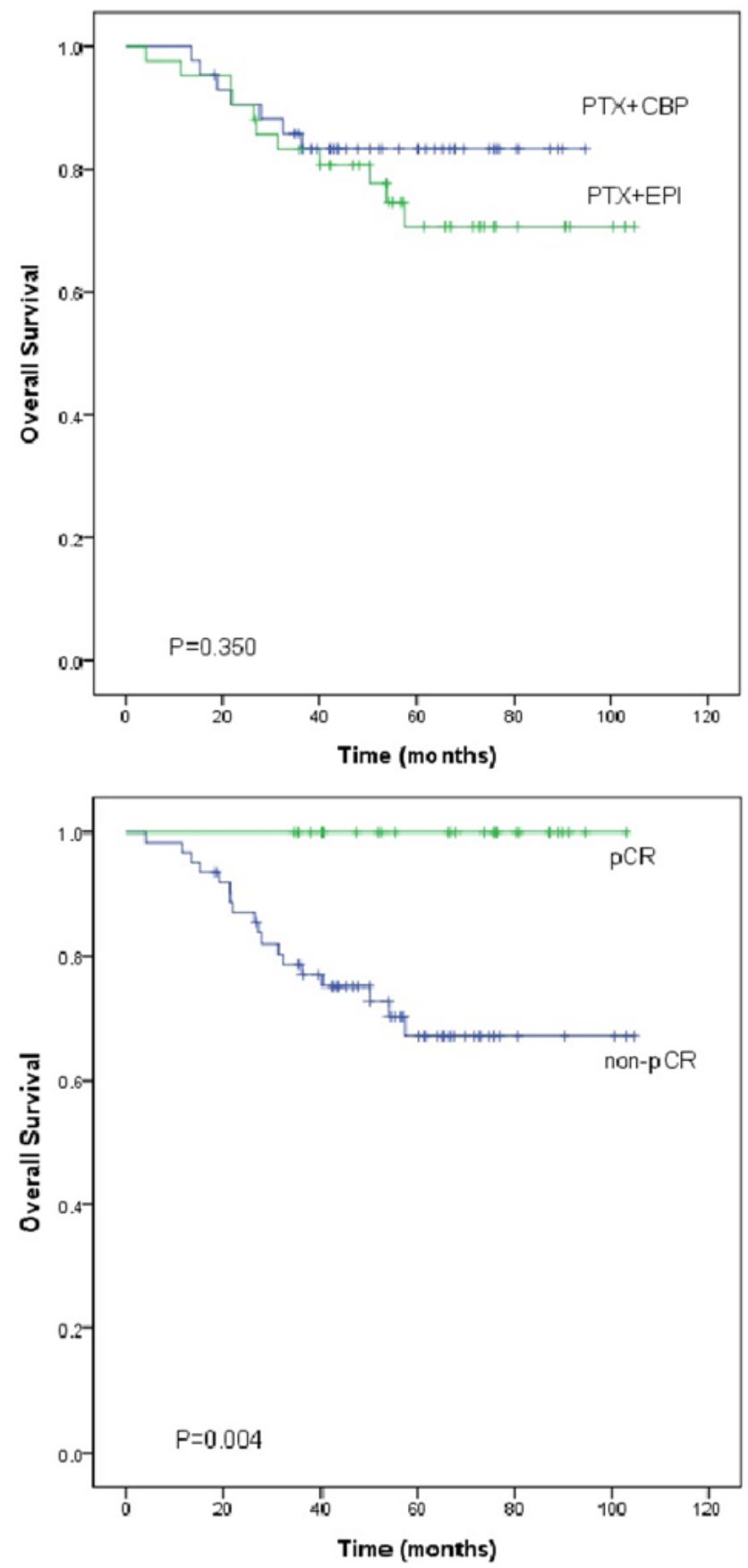

Figure 4: Kaplan-Meier plot of Relapse-free survival (RFS) and overall survival (OS) (a) by different neoadjuvant regimens, and $(b)$ in patients who achieved pathologic complete response (pCR) or not (non-pCR). a. Patients in the paclitaxel plus carboplatin (PTX + CBP) arm had significant better RFS when compared to the epirubicin plus paclitaxel (PTX + EPI) arm $(P$ $=0.043)$. The long-term OS of patients in the PTX $+\mathrm{CBP}$ arm were almost the same as that of patients in the PTX $+\mathrm{EPI}$ arm $(P=0.350)$. $\mathbf{b}$. The pCR patients had significantly better RFS $(P=0.001)$ and OS $(P=0.004)$ than the non-pCR patients. 
biomarkers such as BRCA mutation has been started to further confirm this hypothesis in the near future.

\section{MATERIALS AND METHODS}

\section{Study design and assessments}

This was a prospective, open label, randomized phase II study. TNBC patients were stratified according to clinical stage (II/III), and then randomized to receive PC (paclitaxel plus carboplatin) or EP (epirubicin plus paclitaxel) regimen as NAC. The primary endpoint was $\mathrm{pCR}$ rate, defined as no residual invasive cancer in both excised breast tissue and axillary lymph nodes, or only carcinoma in situ. The secondary endpoints included objective response rate (ORR), RFS, OS and safety.

The clinical evaluation of tumors included physical examination, mammography, ultrasonography of the breast and regional lymph nodes, and breast magnetic resonance imaging (MRI). The clinical or pathological stages were confirmed in accordance with the American Joint Committee on Cancer manual (AJCC, the 6th edition). Clinical responses were assessed every two cycles according to the Response Evaluation Criteria in Solid Tumors (RECIST version 1.0). Adverse effects were defined in accordance with the National Cancer Institute Common Toxicity Criteria for Adverse Events (CTCAE version 3.0). RFS was calculated from the date of randomization to the date of the first local or distant recurrence. OS was defined as the date of randomization to the date of death or last follow-up.

All recruited patients provided written informed consent before treatment as well as verbal consent via telephone for the collection of information from their medical record. The protocol was approved by the Institutional Review and Ethics Board of Cancer Institute/ Hospital of the Chinese Academy of Medical Sciences. The trial is registered in Clinical Trials. Gov (Trial registration ID: NCT01276769).

\section{Patient selection and treatment}

Major eligibility criteria included: 1) women aged 18-75 years; 2) ECOG score 0-1;3) pathologically confirmed breast invasive ductal cancer by core needle biopsy, ER/PR/Her-2 negative by immunohistochemistry (IHC), 4) clinical stage IIA-IIIC with NAC indication; 5) measurable lesions; 6) normal cardiac, hepatic and marrow function. Patients were excluded if they had a history of invasive cancer or prior exposure to chemotherapy/ radiotherapy.

The PC regimen consisted of paclitaxel $175 \mathrm{mg} / \mathrm{m}^{2}$ on day 1 plus carboplatin Area Under the Curve (AUC) $=5$ on day 2, both administered via intravenous infusion (IV), every 3 weeks for $4-6$ cycles. The EP regimen consisted of epirubicin $75 \mathrm{mg} / \mathrm{m}^{2}$ on day 1 and paclitaxel $175 \mathrm{mg} / \mathrm{m}^{2}$ on day 2, both IV, every 3 weeks for 4-6 cycles.

Patients underwent modified radical mastectomy or breast-conserving surgery within four weeks from the last NAC cycle. Patients who did not complete six cycles of NAC received postoperative chemotherapy. For those who achieved objective response before surgery, additional chemotherapy with the same regime as NAC was administered, with a total of six cycles of perioperative chemotherapy. The rest of patients received alternative adjuvant chemotherapy regimens other than those used in NAC. Postoperative radiation was at the discretion of the treating physicians in accordance with guidelines.

\section{Tumor tissue assessment}

ER and PR were defined as negative when $<10 \%$ of nuclei were positively stained in ten high-power fields. Her- 2 was considered negative if IHC scoring was $0 / 1+$, or $2+$ but FISH negative. Cytokeratin 5/6 (CK5/6), epidermal growth factor receptor (EGFR) and $\mathrm{Ki}-67$ status were assessed by IHC. CK5/6 or EGFR was considered positive if any of the invasive tumors cells showed nuclear staining or membrane staining.

\section{Sample size justification}

The sample size calculation is based on the primary endpoint, i.e. pCR rate. Assuming a rate difference of 0.26 between the two regimens (result of preliminary experiments), a single stage design results in a sample size of at least 42 patients for each arm with a 2-sided type I error rate 0.05 and power 0.8 .

\section{Statistical analysis}

All statistical analyses were conducted with SPSS 18.0. Fisher's exact test was used to compare pCR rate, ORR and safety profile between the two regimens. RFS and OS were estimated by the Kaplan-Meier method and compared by log-rank test. Subgroup analysis was performed for the following categorical variables: age, menopause status, tumor size, number of lymph nodes and tumor stage. Risk ratio and its $95 \%$ confidence interval were estimated using Mantel-Haenszel method and a forest plot was created using Review Manage 5.2 (http:// handbook.cochrane.org/).

\section{CONFLICTS OF INTEREST}

The authors have declared no conflicts of interest. 


\section{GRANT SUPPORT}

This study was supported by grant from Cancer Hospital, Chinese Academy of Medical Sciences (LC2010A03).

\section{REFERENCES}

1. Bauer KR, Brown M, Cress RD, Parise CA, Caggiano V. Descriptive analysis of estrogen receptor (ER)-negative, progesterone receptor (PR)-negative, and HER2-negative invasive breast cancer, the so-called triple-negative phenotype: a population-based study from the California cancer Registry. Cancer. 2007; 109: 1721-8. doi: 10.1002/ cncr.22618.

2. Foulkes WD, Smith IE, Reis-Filho JS. Triple-negative breast cancer. N Engl J Med. 2010; 363: 1938-48. doi: 10.1056/NEJMra1001389.

3. Liedtke C, Mazouni C, Hess KR, Andre F, Tordai A, Mejia JA, Symmans WF, Gonzalez-Angulo AM, Hennessy B, Green M, Cristofanilli M, Hortobagyi GN, Pusztai L. Response to neoadjuvant therapy and long-term survival in patients with triple-negative breast cancer. J Clin Oncol. 2008; 26: 1275-81. doi: 10.1200/jco.2007.14.4147.

4. Huober J, von Minckwitz G, Denkert C, Tesch H, Weiss E, Zahm DM, Belau A, Khandan F, Hauschild M, Thomssen C, Hogel B, Darb-Esfahani S, Mehta K, et al. Effect of neoadjuvant anthracycline-taxane-based chemotherapy in different biological breast cancer phenotypes: overall results from the GeparTrio study. Breast Cancer Res Treat. 2010; 124: 133-40. doi: 10.1007/s10549-010-1103-9.

5. Haffty BG, Yang Q, Reiss M, Kearney T, Higgins SA, Weidhaas J, Harris L, Hait W, Toppmeyer D. Locoregional relapse and distant metastasis in conservatively managed triple negative early-stage breast cancer. J Clin Oncol. 2006; 24: 5652-7. doi: 10.1200/jco.2006.06.5664.

6. Cortazar P, Zhang L, Untch M, Mehta K, Costantino JP, Wolmark N, Bonnefoi H, Cameron D, Gianni L, Valagussa P, Swain SM, Prowell T, Loibl S, et al. Pathological complete response and long-term clinical benefit in breast cancer: the CTNeoBC pooled analysis. Lancet. 2014; 384: 164-72. doi: 10.1016/s0140-6736(13)62422-8.

7. Giacchetti S, Porcher R, Lehmann-Che J, Hamy AS, de Roquancourt A, Cuvier C, Cottu PH, Bertheau P, Albiter M, Bouhidel F, Coussy F, Extra JM, Marty M, et al. Long-term survival of advanced triple-negative breast cancers with a dose-intense cyclophosphamide/anthracycline neoadjuvant regimen. Br J Cancer. 2014; 110: 1413-9. doi: 10.1038/ bjc.2014.81.

8. Sikov WM, Berry DA, Perou CM, Singh B, Cirrincione CT, Tolaney SM, Kuzma CS, Pluard TJ, Somlo G, Port ER, Golshan M, Bellon JR, Collyar D, et al. Impact of the addition of carboplatin and/or bevacizumab to neoadjuvant once-per-week paclitaxel followed by dose-dense doxorubicin and cyclophosphamide on pathologic complete response rates in stage II to III triple-negative breast cancer: CALGB 40603 (Alliance). J Clin Oncol. 2015; 33: 13-21. doi: $10.1200 /$ jco.2014.57.0572.

9. von Minckwitz G, Schneeweiss A, Loibl S, Salat C, Denkert C, Rezai M, Blohmer JU, Jackisch C, Paepke S, Gerber B, Zahm DM, Kummel S, Eidtmann H, et al. Neoadjuvant carboplatin in patients with triple-negative and HER2positive early breast cancer (GeparSixto; GBG 66): a randomised phase 2 trial. Lancet Oncol. 2014; 15: 747-56. doi: 10.1016/s1470-2045(14)70160-3.

10. Hurley J, Reis IM, Rodgers SE, Gomez-Fernandez C, Wright J, Leone JP, Larrieu R, Pegram MD. The use of neoadjuvant platinum-based chemotherapy in locally advanced breast cancer that is triple negative: retrospective analysis of 144 patients. Breast Cancer Res Treat. 2013; 138: 783-94. doi: 10.1007/s10549-013-2497-y.

11. Shinde AM, Zhai J, Yu KW, Frankel P, Yim JH, Luu T, Kruper L, Vito C, Shaw S, Vora NL, Kirschenbaum M, Somlo G. Pathologic complete response rates in triplenegative, HER2-positive, and hormone receptor-positive breast cancers after anthracycline-free neoadjuvant chemotherapy with carboplatin and paclitaxel with or without trastuzumab. Breast. 2015; 24: 18-23. doi: 10.1016/j.breast.2014.10.008.

12. Alba E, Chacon JI, Lluch A, Anton A, Estevez L, Cirauqui B, Carrasco E, Calvo L, Segui MA, Ribelles N, Alvarez R, Sanchez-Munoz A, Sanchez R, et al. A randomized phase II trial of platinum salts in basal-like breast cancer patients in the neoadjuvant setting. Results from the GEICAM/2006-03, multicenter study. Breast Cancer Res Treat. 2012; 136: 487-93. doi: 10.1007/s10549-012-2100-y.

13. Farmer H, McCabe N, Lord CJ, Tutt AN, Johnson DA, Richardson TB, Santarosa M, Dillon KJ, Hickson I, Knights C, Martin NM, Jackson SP, Smith GC, et al. Targeting the DNA repair defect in BRCA mutant cells as a therapeutic strategy. Nature. 2005; 434: 917-21. doi: 10.1038/ nature 03445.

14. Schouten PC, Linn SC. Challenges in the Use of DNA Repair Deficiency As a Biomarker in Breast Cancer. J Clin Oncol. 2015; 33: 1867-9. doi: 10.1200/jco.2014.60.5501.

15. Telli ML, Jensen KC, Vinayak S, Kurian AW, Lipson JA, Flaherty PJ, Timms K, Abkevich V, Schackmann EA, Wapnir IL, Carlson RW, Chang PJ, Sparano JA, et al. Phase II Study of Gemcitabine, Carboplatin, and Iniparib As Neoadjuvant Therapy for Triple-Negative and BRCA1/2 Mutation-Associated Breast Cancer With Assessment of a Tumor-Based Measure of Genomic Instability: PrECOG 0105. J Clin Oncol. 2015; 33: 1895-901. doi: 10.1200/ jco.2014.57.0085.

16. Byrski T, Huzarski T, Dent R, Marczyk E, Jasiowka M, Gronwald J, Jakubowicz J, Cybulski C, Wisniowski R, Godlewski D, Lubinski J, Narod SA. Pathologic complete response to neoadjuvant cisplatin in BRCA1-positive breast cancer patients. Breast Cancer Res Treat. 2014; 147: 401-5. doi: 10.1007/s10549-014-3100-x. 
17. Clavarezza M, Turazza M, Aitini E, Saracchini S, Garrone O, Durando A, De Placido S, Bisagni G, Levaggi A, Bighin C, Restuccia E, Scalamogna R, Galli A, et al. Phase II openlabel study of bevacizumab combined with neoadjuvant anthracycline and taxane therapy for locally advanced breast cancer. Breast. 2013; 22: 470-5. doi: 10.1016/j. breast.2013.03.012.

18. Gonzalez-Angulo AM, Akcakanat A, Liu S, Green MC, Murray JL, Chen H, Palla SL, Koenig KB, Brewster AM, Valero V, Ibrahim NK, Moulder-Thompson S, Litton JK, et al. Open-label randomized clinical trial of standard neoadjuvant chemotherapy with paclitaxel followed by FEC versus the combination of paclitaxel and everolimus followed by FEC in women with triple receptor-negative breast cancerdagger. Ann Oncol. 2014; 25: 1122-7. doi: 10.1093/annonc/mdu124.

19. Livasy CA, Karaca G, Nanda R, Tretiakova MS, Olopade OI, Moore DT, Perou CM. Phenotypic evaluation of the basal-like subtype of invasive breast carcinoma. Mod Pathol. 2006; 19: 264-71. doi: 10.1038/modpathol.3800528.
20. Nielsen TO, Hsu FD, Jensen K, Cheang M, Karaca G, Hu Z, Hernandez-Boussard T, Livasy C, Cowan D, Dressler L, Akslen LA, Ragaz J, Gown AM, et al. Immunohistochemical and clinical characterization of the basal-like subtype of invasive breast carcinoma. Clin Cancer Res. 2004; 10: 5367-74. doi: 10.1158/1078-0432. ccr-04-0220.

21. Carey LA, Perou CM, Livasy CA, Dressler LG, Cowan D, Conway K, Karaca G, Troester MA, Tse CK, Edmiston S, Deming SL, Geradts J, Cheang MC, et al. Race, breast cancer subtypes, and survival in the Carolina Breast Cancer Study. JAMA. 2006; 295: 2492-502. doi: 10.1001/ jama.295.21.2492.

22. Rakha EA, Ellis IO. Triple-negative/basal-like breast cancer: review. Pathology. 2009; 41: 40-7. doi: 10.1080/00313020802563510. 